\title{
Gallium Aluminum Arsenide/Gallium Arsenide Integrated Optical Repeater
}

Bar-Chaim, N, Lau, K, Ury, I, Yariv, A.

N Bar-Chaim, K Y Lau, I Ury, A. Yariv, "Gallium Aluminum Arsenide/Gallium Arsenide Integrated Optical Repeater," Proc. SPIE 0466, Optical Interfaces for Digital Circuits \& Systems, (10 May 1984); doi: 10.1117/12.941564 
Gallium aluminum arsenide/gallium arsenide integrated optical repeater

\author{
N. Bar-Chaim, K.Y. Lau, I. Ury \\ Ortel Corporation \\ 2015 w. Chestnut Street, Alhambra, California 91803 \\ A. Yariv \\ California Institute of Technology, Pasadena, California 91125
}

\title{
Abstract
}

A low threshold buried heterostructure laser, a metal-semiconductor field effect transistor (MESFET), and a photodiode, have for the first time, been monolithically integrated on a semi-insulating GaAs substrate. This integrated optoelectronic circuit (IOEC) was operated as a rudimentary optical repeater. The incident optial signal is detected by the photodiode, amplified by the MESFET, and converted back to light by the laser. The gain bandwidth product of the repeater was measured to be $178 \mathrm{MHz}$.

\section{Introduction}

The goal of integrated optoelectronics is to monolithically integrate optoelectronic components with purely electronic components. In this paper we describe the demonstration of the monolithic integration of a laser, a transistor, and a photodiode. The devices were fabricated on a semi-insulating (SI) GaAs substrate. Use of a SI substrate allows the devices to be electrically insulated from each other, and also reduces parasitics. The simple circuit configuration used serves to illustrate the basic principles of operation of IOECs in general, and sheds light on the expected performance characteristics of more complicated repeater circuits.

\section{Theory of operation}

A schematic circuit of the IOEC is shown in Fig. 1. Light incident on the photodiode causes a current to flow through the load resistor $R_{d}$. The change in current which flows through the laser is the product of the transconciuctance $\left(g_{m}\right)$ of the MESFET and the change in voltage which appears at the transistor gate. The DC optical gain of this repeater is therefore,

$$
\mathrm{G}=\eta_{p d}{ }^{n}{ }_{1}{ }{ }^{R} d
$$

where $n_{p d}$ and $n_{1}$ are the quantum efficiencies of the photodiode and laser (from one facet), respectlvely. The $-3 \mathrm{~dB}$ bandwidth of the repeater is

$$
B=\frac{1}{2 \pi} \quad \frac{1}{R_{d} C}
$$

where $C$ is the combined capacitance of the photodiode and MESFET. The gain-bandwidth (GxB) product of the repeater is, therefore,

$$
G \times B=\frac{{ }^{n} d^{n} 1^{g}}{2 \pi C}
$$

\section{Device fabrication}

A perspective view of the IOEC is shown in Fig. 2. The fabrication steps which were used to realize the device are as follows. Epitaxial layers were grown on a semi-insulating GaAs substrate by means of LPE in the following order: $n$-GaAs layer $\left(\mathrm{n} \simeq 2 \times 10^{16} \mathrm{~cm}^{-3}\right)$, lower cladding layer ( $\left.\mathrm{n}-\mathrm{Ga}_{0.65} \mathrm{~A} \mathrm{l}_{0.35} \mathrm{As}\right)$, active layer (undoped $\left.\mathrm{Ga}_{0} .9{ }_{5} \mathrm{Al} 0.05 \mathrm{As}\right)$, and an upper cladding layer $\left(\mathrm{p}-\mathrm{Ga}_{0} .6{ }_{5} \dot{\mathrm{A}}_{0}\right.$. $\left.{ }_{5} \mathrm{~A} \mathrm{~s}\right)$. The $\mathrm{n}$-GaAs layer performs three functions: it serves as the lower contact layer of the laser, the channel layer of the MESFET, and the photosensitive/contact layer of the photodiode. After epitaxial growth, mesas were etched down to the n-GaAs layer to form the laser waveguides. The width of the active layer thus formed was $\simeq 2 \mu \mathrm{m}$.

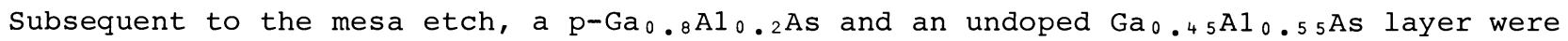
regrown to embed the laser mesas. The $\mathrm{p}-\mathrm{Ga}_{0} .8 \mathrm{~A} \mathrm{I}_{0 .}{ }_{2} \mathrm{As}$ layer performs two functions: It serves as the leakage-current blocking layer of the laser, and as the p-type window layer of the photodiode. The undoped $\mathrm{Ga}_{0.45} \mathrm{~A} 1_{0 .}{ }_{5} \mathrm{As}$ layer was then selectively etched with HCl 
everywhere except in the vicinity of the laser mesa. The exposed p-Ga.0. ${ }_{0} \mathrm{Al}{ }_{0} .{ }_{2} \mathrm{As}$ layer was then etched to define the photodiodes. Mesa etching down to the substrate was used to isolate the devices from each other.

The entire wafer was next coated with $\mathrm{SiO}_{2}$. Windows were etched in the $\mathrm{SiO}_{2}$ to allow contact to be made to the p-type layers of the laser and the photodiode. Zn diffusion was performed to enhance the contact to the GaAlAs. Cr-Au was deposited and etched to form contacts to the anodes of the laser and the photodiode. Contacts to the cathodes of the laser, photodiode, and the MESFET source and drain, were formed by etching through the $\mathrm{SiO}_{2}$, lifting off $\mathrm{AuGe} / \mathrm{Au}$, and alloying. The MESFET gates were formed by etching a recess into the n-GaAs MESFET channel until the desired thickness was obtained, and lifting off Al. The MESFET thus formed had a gate length of $2 \mu \mathrm{m}$ and a gate width of $250 \mu \mathrm{m}$. The size of an individual die was $325 \mu \mathrm{m} \times 625 \mu \mathrm{m}$.

\section{Device performance}

The light vs current characteristic of the laser is shown in Figure 3 . The threshold current of 15-20 mA is typical of this laser structure. The transconductance characteristics of the FET are shown in Fig. 4, where the drain current is plotted against the voltage across the series combination of the laser and the FET, at various gate voltages. One interesting observation is that as the gate voltage is decreased from 0 to $-8 \mathrm{~V}$, the drain current decreases continuously, but with a slight further decrease in the gate voltage (to -8.03V) the FET is completely cut-off and no current passes. The FET remains in the cut-off state unless the gate voltage is raised above $-7.7 \mathrm{~V}$, at which point the transistor resumes its normal transconductance characteristic. It is believed that this bistability is due to the coupling of the laser emission into the channel of the FET, which is sensitive to optical radiation. An increase in the gate voltage increases the drain current, and, hence, the amount of laser emission, which decreases the resistance of the transistor channel and consequently further increases the drain current. This positive feedback effect can lead to a bistable characteristic as that observed in our devices.

The operation of our IOEC as a repeater was demonstrated using the circuit arrangement shown in Fig. 1. The load resistance $R_{d}$ shown in Fig. 1 was a chip resistor mounted next to the device. Laser emission from a separate GaAlAs laser diode is focused by a $20 x$ objective on the photodiode in the IOEC. The absolute DC responsivity of the photodiode was measured separately to be $0.45 \mathrm{~A} / \mathrm{W}$. The absolute amount of light falling on the detector can thus be determined by monitoring the DC photocurrent through $R_{d}$. The DC optical gain $G$ was measured to be $15 \mathrm{~dB}$ with $\mathrm{R}_{\mathrm{d}}=5.4 \Omega$, and $9.2 \mathrm{~dB}$ with $\mathrm{R}_{\mathrm{d}}=2.7 \mathrm{k} \Omega$. This is in good agreement with that expected by putting in the measured values of $n_{\mathrm{pd}}(0.45 \mathrm{~A} / \mathrm{W})$, $\mathrm{G}_{\mathrm{m}}(6 \mathrm{mmho})$ and $n_{1}(0.4 \mathrm{~W} / \mathrm{A})$ into $\mathrm{Eq}$. (1). The bandwidth of the repeater was measured by modulating the source laser and observing on a RF spectrum analyzer the modulation in the laser output from the IOEC with a separate PIN detector. The combined frequency response of the external laser and detector was calibrated by repeating the measurement with the IOEC removed and the optical output from the external laser was directly detected by the external photodetector. The calibrated frequency responses of the repeater are shown in Fig. 5. The $-3 \mathrm{~dB}$ bandwidths are $29 \mathrm{MHz}$ with $\mathrm{R}_{\mathrm{d}}=5.4 \Omega$ and $52 \mathrm{MHz}$ with $\mathrm{R}_{\mathrm{d}}=2.7 \mathrm{k} \Omega$. The high-frequency fall-off is at approximately $20 \mathrm{~dB} / \mathrm{dec}$, indicating that the response is manifested by a simple RC combination. The observed bandwidth indicates a value of $C$ in the vicinity of $1 \mathrm{pF}$ according to Eq. (2). This value is consistent with that obtained by separate measurements ${ }^{2}$ and is within the range of values generally predicted for these kind of devices. The measured gain-bandwidth product was $178 \mathrm{MHz}$.

\section{Conclusion}

The above results illustrate clearly the operational principle of an integrated optoelectronic repeater circuit. The simple single-transistor circuit described above has a GxB product of $178 \mathrm{MHz}$. It is obvious from Eq. (2), that GxB can be increased by improvement in the performance of the individual components in the IOEC (increasing $\eta_{p d}, \eta_{l}, g_{m}$, and decreasing C). However, it should be noted that the laser and detector in our repeater already display state-of-the-art performance. Nevertheless, an increase of a factor of two in $n_{1}$ can be obtained by reflection-coating the rear facet of the laser. The transconductance of the FET can also be improved by reducing the gate-length to sub-micron dimensions. With these improvements, the GxB product of our device would exceed 1 GHz. This is the limit to the performance of a basic single-transistor repeater circuit. Achieving higher gain-bandwidth products would require more complicated IOEC circuits containing multiple stage transistor amplifiers.

\section{Acknowledgement}

This work was supported by the Defense Advanced Research Project Agency and the Naval Research Laboratory. 
1. N. Bar-Chaim, I. Ury and A. Yariv, IEEE spectrum, 19, 38 (1982), and references contained therein.

2. N. Bar-Chaim, K.Y. Lau, I. Ury and A. Yariv, Appl. Phys. Lett., 43, 261 (1983).

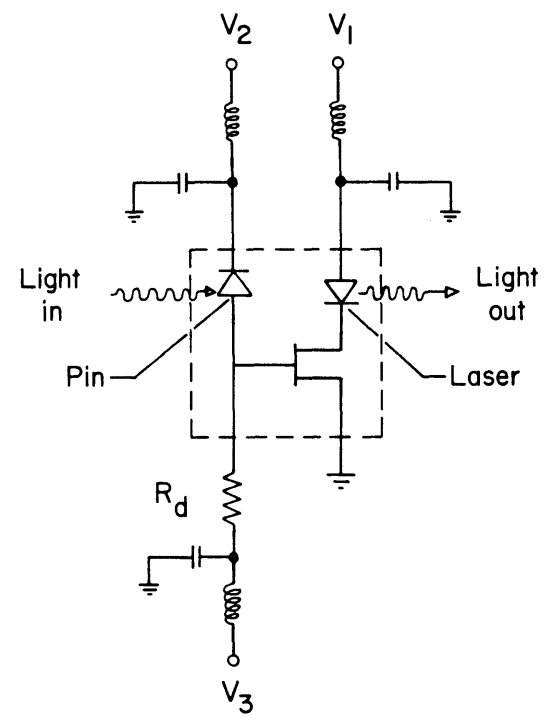

Figure 1. Schematic diagram of the IOEC repeater. Also shown are the bias networks for DC and RF testing of the circuit.

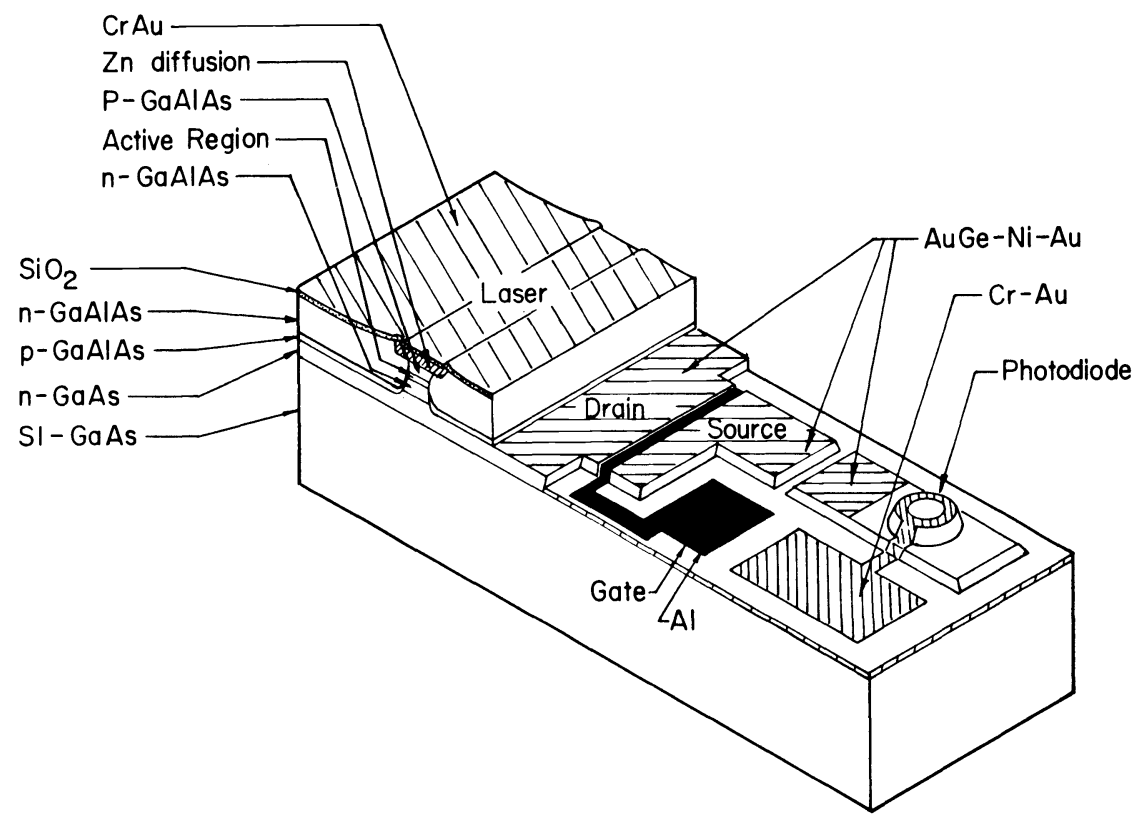

Figure 2. A perspective view of the IOEC. 


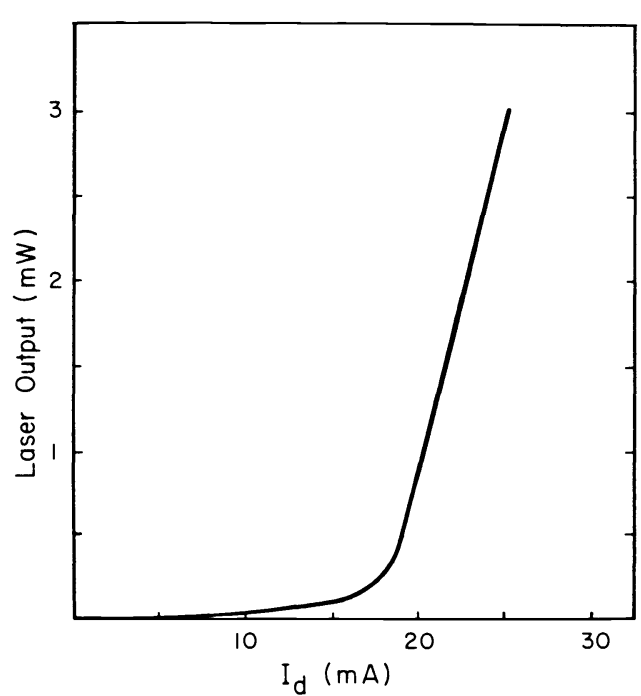

Figure 3. Light vs. current characteristics of the laser in the IOEC.

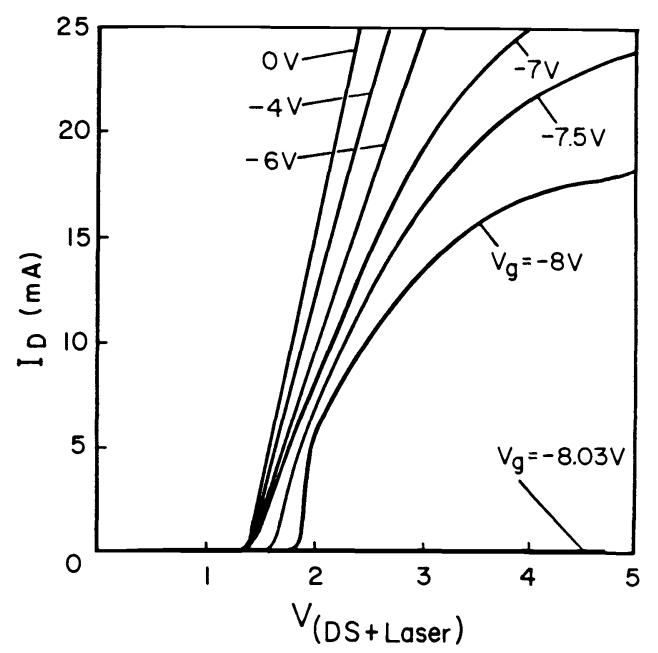

Figure 4. I-V characteristics of the MESFET at various gate voltages. Note the sudden total pinch-off at $\mathrm{V}_{\mathrm{g}}=-8.03 \mathrm{~V}$. The channel does not recover until $\mathrm{V}_{\mathrm{g}}$ is increased to above $-7.7 \mathrm{~V}$.

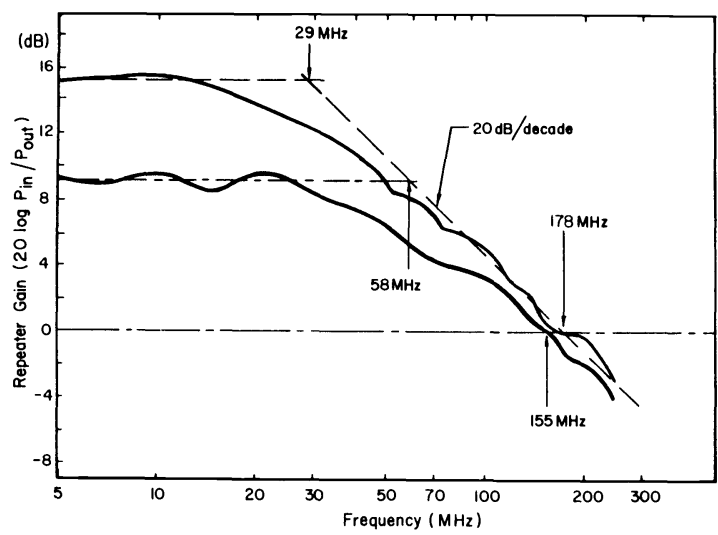

Figure 5. Frequency response of the repeater unit at two values of the bias resistor $\mathrm{R}_{\mathrm{d}}$. Top curve: $\mathrm{R}_{\mathrm{d}}=5.4 \Omega$, lower curve: $R_{\mathrm{d}}=2.7 \mathrm{k} \Omega$. 